\title{
Laboreal
}

Volume 17 №2 | 2021

Trabalho e Território

\section{La realidad de la actividad de trabajo en el proceso de transformación de los productos del mar : cuando el territorio determina inequidades}

A realidade da atividade de trabalho no processo de transformação de produtos do mar: quando o território determina inequidades

La réalité de l'activité de travail dans le processus de transformation des produits de la mer: quand le territoire détermine les inégalités The reality of the work activity in the process of transformation of sea products: when the territory determines inequalities

\section{Pamela Astudillo, Carlos Ibarra y Francisco Valdés}

\section{OpenEdition}

\section{Journals}

\section{Edición electrónica}

URL: https://journals.openedition.org/laboreal/18617

DOI: $10.4000 /$ laboreal. 18617

ISSN: 1646-5237

Editor

Universidade do Porto

Referencia electrónica

Pamela Astudillo, Carlos Ibarra y Francisco Valdés, «La realidad de la actividad de trabajo en el proceso de transformación de los productos del mar : cuando el territorio determina inequidades», Laboreal [En línea], Volume $17 N^{\circ} 2$ | 2021, Publicado el 03 diciembre 2021, consultado el 05 diciembre 2021. URL: http://journals.openedition.org/laboreal/18617; DOI: https://doi.org/10.4000/laboreal. 18617

Este documento fue generado automáticamente el 5 diciembre 2021.

Laboreal está licenciado com uma Licença Creative Commons - Atribuição-NãoComercial 4.0 Internacional. 


\section{La realidad de la actividad de trabajo en el proceso de transformación de los productos del mar : cuando el territorio determina inequidades}

A realidade da atividade de trabalho no processo de transformação de produtos do mar: quando o território determina inequidades

La réalité de l'activité de travail dans le processus de transformation des produits de la mer: quand le territoire détermine les inégalités The reality of the work activity in the process of transformation of sea products: when the territory determines inequalities

Pamela Astudillo, Carlos Ibarra y Francisco Valdés

\section{NOTA DEL EDITOR}

Manuscrito recibido en : 07/06/2021

Aceptado tras peritaje : 13/09/2021

\section{Introducción}

La actividad de la pesca, sin dudas tiene un arraigo territorial en todo Chile, dado sus más de $4.000 \mathrm{~km}$ de costa, con emplazamientos industriales en prácticamente todas las regiones del país. En esta industria, la actividad de trabajo produce identificación y conocimientos del oficio a partir de un contexto dado, que no es posible encontrar en otro territorio (Major \& Vézina, 2011, 2015). El procesamiento de productos del mar, 
involucra formas de trabajo artesanales, realizadas desde sus inicios de una manera en que, por lo general, se encuentra en ausencia de acciones que favorezcan la seguridad y la salud de trabajadoras y trabajadores, ignorando muchas veces la eficacia en los procesos y el aseguramiento de la calidad en los productos (Major \& Vézina, 2015).

2 La Fuerza de Trabajo Ocupada en Chile es de aproximadamente 8.500.000 trabajadores, y sólo un $70 \%$ de ellos se encuentra dentro del empleo formal, con contrato de trabajo y cobertura de protección social siendo la tasa de participación en el empleo de un $71 \%$ para los hombres y un $45 \%$ para las mujeres (Instituto Nacional de Estadísticas, 2021). Existe, además, una estructura del empleo formal marcada por la división sexual del trabajo, en sentido vertical y horizontal en la mayor parte de la industria, notablemente a causa de la influencia del taylorismo (Astudillo \& Ibarra, 2014). Dentro de la actividad económica de la pesca existe un $93 \%$ de ocupación masculina y un $7 \%$ femenina (INE, 2021). Habitualmente, y a causa de la división sexual del trabajo, en este sector como en otros de la industria agroalimentaria, las actividades minuciosas son realizadas por las mujeres, teniendo un tipo de contrato a trato y/o temporal y las actividades como la manipulación de cargas que se realiza al inicio o al final de las líneas de producción es realizado por los hombres, como también aquellas actividades previas al procesamiento de los productos, particularmente en la pesca en el mar (Messing, 2000 ; Messing, Lefrançois, \& Saint-Charles, 2018).

3 Si bien es cierto que el trabajo es feminizado dentro de las plantas de procesamiento de productos del mar, no es común encontrar estudios o intervenciones que analicen el trabajo bajo la perspectiva de género (Astudillo \& Ibarra, 2014), ni en cómo comprender el fenómeno del trabajo en estas trabajadoras para identificar las acciones que ayudarán a realizar el plan de mejoras. Tampoco el estudio de los trabajadores eventuales (trabajadores por día o temporada) ha sido objeto de estudios ergonómicos, siendo estas actividades realizadas en contextos masculinizados (Astudillo \& Ibarra, 2014). Por otra parte, el concepto de "territorio" no ha sido explorado en los estudios ergonómicos, aun cuando este puede representar un importante determinante de la actividad de trabajo, particularmente en este sector que representa una fuente de trabajo importante en Chile y que tiene particularidades propias en cada región de norte a sur, por los aspectos climáticos característicos por el desierto por el norte y la cercanía a la antártica por el sur.

4 Un aspecto común en los trabajos en el sector pesquero es la predominancia del trabajo manual y físico, en ese marco los trastornos músculo-esqueléticos (TME) representan un problema de salud importante para la industria agroalimentaria de Chile (Ibarra \& Astudillo, 2021), particularmente para el sector de la industria pesquera que según datos de la Superintendencia de Seguridad Social (SUSESO) en Chile, generan mayores prevalencias de TME en las regiones del sur de Chile por la mayor presencia de plantas faenadoras de salmón con un $20 \%$ del total de las enfermedades profesionales en esas regiones (SUSESO, 2020). Esto ha llevado a una necesidad de implementar dispositivos de prevención y evaluaciones de riesgos ligadas a las regulaciones del sector sanitario, lo que obliga a las empresas buscar la asesoría en ergonomía con las aseguradoras (mutualidades), las cuales se basan en la aplicación listas de chequeo que derivan de las normas ISO 11.228, y que apoyan la presentación de las medidas y mejoras de las condiciones en la empresa, con resultados variados dependiendo de los niveles de experiencia y formación de los ergónomos y profesionales de SST, con resultados muy variables (Castellucci et. al., 2021). 
5 Teniendo en cuenta que la intervención ergonómica "es realizada dentro de un contexto dado, en un momento dado, en vista de transformar las situaciones de trabajo para mejorar según los criterios de salud y eficacia. La intervención implica llevar a cabo un sistema organizado de acciones, en interacción con los actores de la empresa" (St. Vincent et al., 2011, p. 64), es que en este trabajo se mostrarán dos diagnósticos ergonómicos realizados para contribuir a mejorar los procesos productivos sobre la actividad de trabajo de las trabajadoras(es), tanto para una empresa que realiza la transformación de productos del mar, como en otra donde los trabajadores descargan el pescado desde las embarcaciones para la producción harina de pescado, a fin de indagar en la actividad de mujeres en un puesto de trabajo feminizado (más de un $50 \%$ mujeres) y los hombres en un contexto masculinizado, los determinantes de su actividad de trabajo y la influencia del territorio que es compartido en ambos contextos. El análisis de la actividad como aproximación para comprender el trabajo será central, ya que permitirá mostrar la problemática de salud en el trabajo en hombres y mujeres frente a los riesgos en la actividad y demostrar las diferencias de género en el análisis de las actividades productivas, las que serán descritas desde el proceso de las intervenciones ergonómicas realizadas en dichas empresas.

El objetivo de la presente contribución, es mostrar a través del análisis de la actividad en dos estudios de caso, la actividad real de trabajo en dos empresas, una de ella es el eviscerado de los ostiones, como puesto feminizado en una empresa procesadora de ostiones, y en el segundo caso, es el trabajo de los operadores de descarga de pescado, desde las bodegas de la embarcación, como puesto masculinizado en una segunda empresa. Ambas empresas comparten el mismo contetxo territorio y sector de actividad productiva.

\section{Metodología}

7 El presente artículo se basa en 2 estudios de caso, ambos con aproximación mixta. El cuadro teórico se basa en el modelo centrado en la persona en actividad (St-Vincent et al., 2011) que permite comprender la situación laboral, considerando los determinantes y las exigencias de la actividad de trabajo, el margen de maniobra y los efectos sobre la salud del trabajador/a. Ambos estudios se llevaron a cabo mediante el esquema de la intervención ergonómica (Gúerin et al., 2007), considerando acciones planificadas y sistematizadas. En ambos se seleccionó, para fines de este artículo, las siguientes etapas de la intervención : 1) Análisis de la demanda, 2) Prediagnóstico y 3) Propuesta de la intervención ergonómica.

Los 2 casos cuentan con observaciones abiertas y sistemáticas en diferentes horarios tanto diurnos como nocturno, incluyendo un protocolo de observación sistemático con el software ActoGraph ${ }^{\circledast}$ para analizar las posturas en función de la actividad de trabajo (caso 2). La percepción de dolencias osteomusculares se recabó con un esquema corporal (Vézina et al., 2009) y la Escala de percepción del esfuerzo de Borg-10 modificada. Se realizaron entrevistas semiestructuradas confeccionando una entrevista tipo, además de las verbalizaciones simultáneas.

9 Para efectos de esta comunicación se abordarán a modo de comparación las etapas de : 1. Análisis de la demanda ; 2. Prediagnóstico ; y 3. Propuestas de soluciones. El análisis de la demanda tiene en cuenta el contexto para llegar a un mandato, el Prediagnóstico es llevado a cabo mediante investigaciones preliminares, acerca del contexto, las 
personas, la actividad, etc. A partir del cual se obtienen resultados preliminares y que sirven para conocer cuáles son los determinantes de las actividades de trabajo estudiadas, mayor detalle de los tiempos dedicados en Tabla 1.

Tabla 1 : Tiempo destinado a observaciones, entrevistas, verbalizaciones y filmación para los casos

\begin{tabular}{|c|c|c|}
\hline Tipo de registro & $\begin{array}{c}\text { CASO } 1 \\
\text { Trabajadoras de eviscerado } \\
\text { de los ostiones, en planta } \\
\text { procesadora } \\
\text { (Empresa 1) }\end{array}$ & $\begin{array}{c}\text { CASO } 2 \\
\text { Operadores de descarga } \\
\text { de pescado desde las } \\
\text { bodegas de una embarcación } \\
\text { (Empresa 2) }\end{array}$ \\
\hline Número de Trabajadores & 39 Mujeres & 6 Hombres \\
\hline $\begin{array}{l}\text { Observación abiertas y } \\
\text { sistemáticas }\end{array}$ & $26 \mathrm{~h}$ & $20 \mathrm{~h}$ \\
\hline Filmación de videos & $6 \mathrm{~h}$ & $4 \mathrm{~h}$ \\
\hline Entrevistas y verbalizaciones & $8 \mathrm{~h}$ & $3 \mathrm{~h}$ \\
\hline \multicolumn{3}{|c|}{$\begin{array}{l}\text { Tabla 1: Tiempo destinado a observaciones, entrevistas, verbalizaciones y filmación para los dos } \\
\text { casos. }\end{array}$} \\
\hline
\end{tabular}

Tabla 1 : Tiempo destinado a observaciones, entrevistas, verbalizaciones y filmación para los casos

\section{Resultados}

\subsection{Caso 1 : Trabajadoras de eviscerado de los ostiones, en planta procesadora}

\subsubsection{Contexto, demanda inicial y actividad}

10 La empresa donde se realiza el estudio lleva más de 30 años en el mercado nacional e internacional, exportando a países de Europa como de Asia, tiene plantas de procesamiento de productos del mar en gran parte del territorio nacional a lo largo de los $4000 \mathrm{~km}$ de costa que tiene el país. La demanda inicial surge desde el Departamento de Prevención de Riesgos, este solicita "Realizar una intervención ergonómica para disminuir el número elevado de accidentes y de trastornos músculo-esqueléticos (TME) en los puestos de desconchado y de evisceración de ostiones".

11 En general, el proceso de transformación del ostión, se inicia con la etapa de recepción de los ostiones provenientes de diferentes zonas de cultivo, en la bahía de la ciudad donde esta se emplaza y corresponde a zonas concesionadas por el Estado de Chile a las empresas para su explotación controlada, luego se realiza el Escaldado donde los ostiones experimentan un shock térmico, pasan luego a la etapa de Desconchado donde se quitan las conchas y luego a la sección de Eviscerado, en la cual se quitan las vísceras del ostión y se realiza el lavado, para posteriormente continuar el proceso hasta su almacenamiento en la cámara de frío. 
12 En la sección de Eviscerado existen diferentes presentaciones comerciales del producto, según el calibre del ostión, según el mercado al cual va dirigido, la variedad, existen ostiones media concha o sin concha, es en el ostión sin concha en la cual se concentrará la descripción. En el puesto de eviscerado, la trabajadora debe completar su trabajo según una meta de producción personal, en la cual debe realizar al menos 12 bol (6 Kg cada uno) para acceder a la escala de bonificación.

13 El macro ciclo de 1 bol dura aproximadamente 30 minutos y cada micro ciclo, que corresponde a la evisceración de 1 solo ostión, dura aproximadamente 4 segundos. A continuación, se describen las operaciones que realiza la trabajadora en un micro ciclo al eviscerar 1 ostión :

1. Mira la canasta y calcula el tamaño de los ostiones.

2. Calcula el tiempo que le llevará completar su trabajo.

3. Toma y acomoda el ostión entre los dedos de su mano.

4. Usa el cuchillo con la mano dominante

5. Tirar del manto del ostión con el cuchillo.

6. Hace un corte en la cabeza del ostión, al mismo tiempo, que sostiene la base del ostión con la otra mano.

7. Envuelve el manto en la hoja del cuchillo.

8. Tira de las vísceras con la ayuda del cuchillo para sacarlas. Simultáneamente con la mano opuesta, gira el mango para ayudar a tirar de las vísceras.

9. Con la mano sosteniendo el cuchillo, arroja las vísceras al contenedor de desechos. Al mismo tiempo, observa si todavía hay desperdicio.

10. Si es necesario, raspa con el cuchillo el contorno del ostión para sacar desechos

11. Lanza el ostión en el recipiente, sujetando el cuchillo. Al mismo tiempo, observa el próximo ostión que va eviscerar. 
Figura 1 : Trabajadores eviscerando ostiones

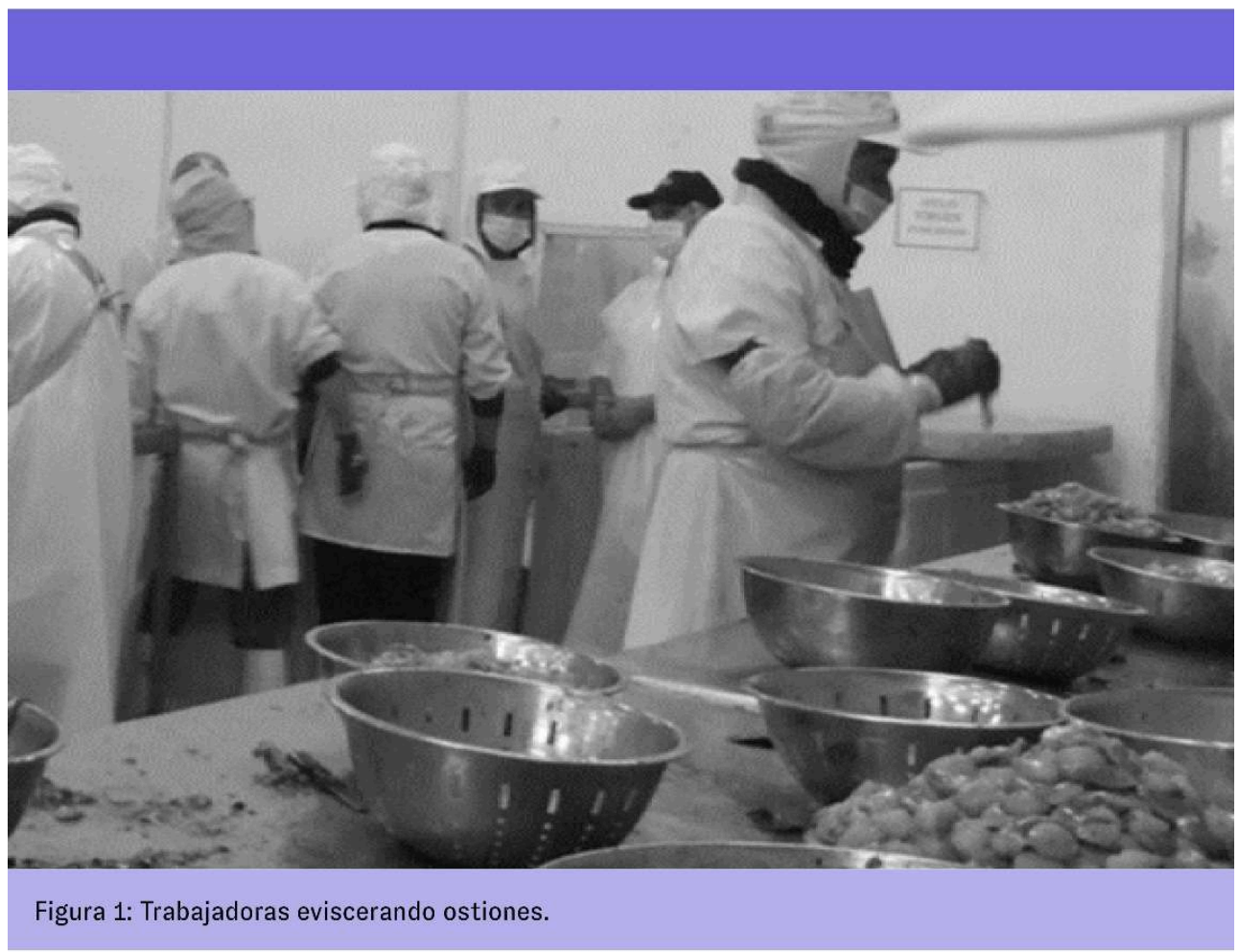

Figura 1 : Trabajadores eviscerando ostiones

14 La trabajadora de evisceración debe tener en cuenta retirar las vísceras, evitar sacar el coral (gónada), tallar o cortar el ostión, lo cual implica obtener un producto de menor valor que irá al desperdicio.

\subsubsection{Prediagnóstico}

Efectos detectados: Los síntomas de dolor osteomuscular fueron importantes en las trabajadoras de eviscerado. Las zonas más frecuentes de dolor referidas por las trabajadoras fueron la espalda baja zona lumbar (41\%), Hombros $(35,9 \%)$, brazos $(30,8 \%)$, muñecas $(23,1 \%)$, Dorso $(23,1 \%)$ y piernas $(15,4 \%)$ entre otras, más detalle en la Figura 2. 
Figura 2 : Frecuencia porcentual del dolor referido por las trabajadoras de eviscerado (Empresa $\mathbf{i}$

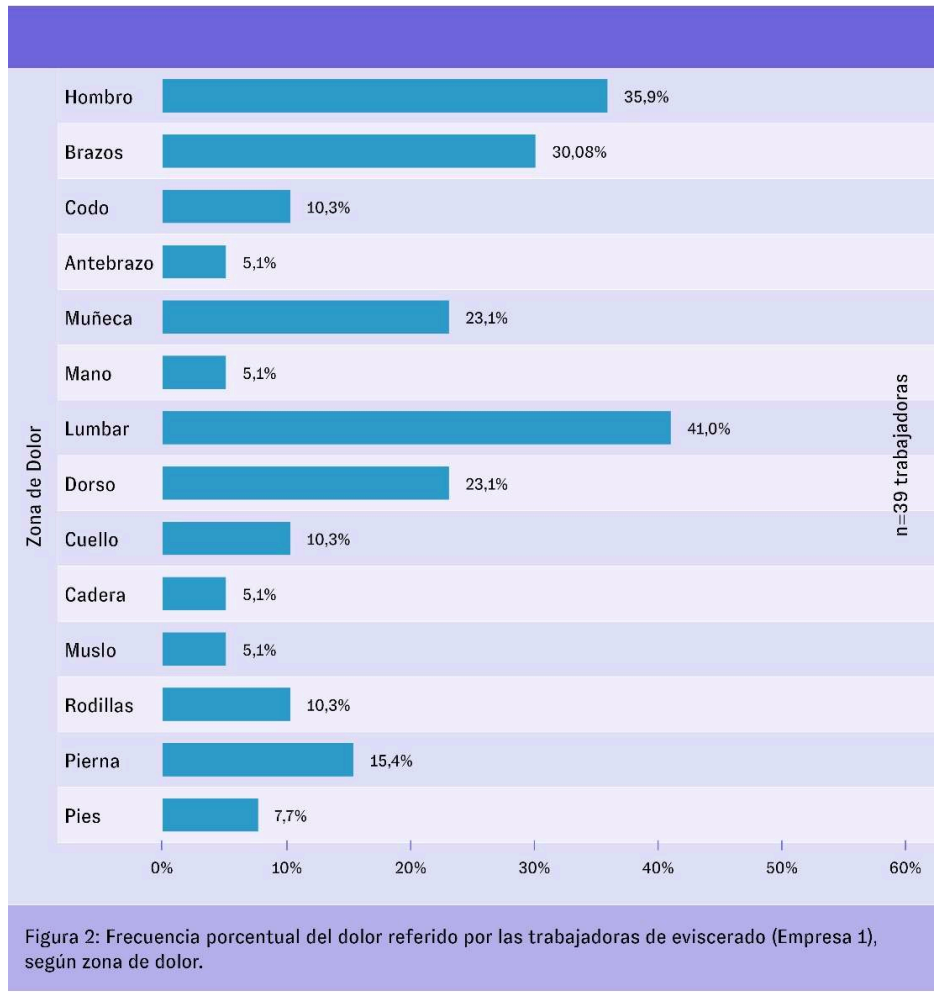

Figura 2 : Frecuencia porcentual del dolor referido por las trabajadoras de eviscerado (Empresa i

Se demostró que las trabajadoras están expuestas a diversas operaciones exigentes, siendo por ellas con mayor frecuencia asociado al dolor, levantar la canasta con un $48 \%$, el trabajo de pie un $21 \%$ y la repetitividad de la actividad de evisceración con un $10 \%$. Los problemas de calidad están asociadas a la pérdida de producto, aumento en los tiempos de producción, elevado ausentismo y rotación de personal.

Variabilidad del producto : El ciclo productivo del ostión dura de 16 a 20 meses, desde el momento en que se obtiene la semilla hasta su extracción. La materia prima proviene de la bahía cercana a $6 \mathrm{~km}$ de la planta, pero también desde otra región que se encuentra a $450 \mathrm{~km}$. Las trabajadoras, prefieren los productos que vienen desde la otra región, porque es más turgente y fácil de manipular. A veces, la materia prima llega a la antigua planta de proceso y los ostiones se ponen más duros y complicados de abrir, según los trabajadores de la sección de desconche. Hay veces que el transporte se retrasa y que las trabajadoras tienen que repartirse la materia prima para alcanzar su producción.

En síntesis, hay tres tipos de producto que se elaboran en la fábrica : ostión IQF, ostión fresco refrigerado y ostión media concha. El más solicitado por el cliente es el IQF. Las trabajadoras de la evisceración dicen que para ellas es más fácil y menos restrictivo hacer ostión IQF, dependiendo de la técnica de evisceración. Con el ostión IQF pueden ellas alcanzar las escalas de bonificación más rápidamente. Por otro lado, hay trabajadoras que dicen que el ostión IQF es más cómodo trabajarlo, porque es más grande y no resbala tanto, ya que en cuanto al agarre son diferentes. Contrariamente, trabajar con ostión de media concha es más difícil, porque las conchas presionan su 
mano y les genera dolor, además no pueden hacer todos los kilos que quieren para ganar más dinero, ya que los pedidos son de menor volumen.

Problemas de calidad : Las trabajadoras refieren que hay cuellos de botella aguas arriba, cuando hay pocos trabajadores en la sección de desconchado. Esto implica que los ostiones de producción IQF no están listos para el siguiente proceso, que es la evisceración. Además, los trabajadores del puesto de desconchado, tienen un cuello de botella aguas arriba también porque el camión que distribuye, se retrasa en llegar a la planta de proceso desde los centros de cultivo de ostiones. Para los trabajadores esto representa una pérdida de tiempo y dinero, lo que los lleva a trabajar más rápido, para alcanzar las metas de producción y lograr los objetivos de producción individuales.

Aspectos colectivos del trabajo : El trabajo en esta sección es individual, sin embargo, hay ocasiones en las que las trabajadoras tienen actividad grupal para lograr sus objetivos de producción, hay algunas trabajadoras que muestran una actitud más colaborativa, pero no es la generalidad.

21 En el aspecto social, a veces comparten una bandeja entre dos trabajadoras o entre las trabajadoras que están eviscerando en el mismo mesón, esto ocurre cuando hay una disminución de producto. Sin embargo, cuando hay una disminución más acentuada en la producción aguas arriba, tienen que hacer fila para a recibir el producto, lo hace que compitan entre ellas por la cantidad de ostiones disponibles, generándose conflictos entre las trabajadoras.

\subsubsection{Descripción de las limitaciones, exigencias y riesgos asociados a la actividad de eviscerado}

Dimensión física: Es una exigencia importante en la actividad, asociada a la repetitividad y la falta de rotación, lo que produce exposición a riesgo de TME. También la repetición en el traslado de los bols y las bandejas con ostiones es una exigencia importante, lo que implica el uso de fuerza al tomar, levantar y trasladar hacia el mesón de trabajo, pasando por un piso húmedo, que conlleva el riesgo de caídas. Por otra parte, el trabajo es exigente en cuanto a la precisión de los gestos, ya que de esta habilidad depende su producción individual y la calidad del producto, hay esfuerzos de agarre en pinza, lo que implica desviación lateral, flexión y extensión de la muñeca de manera repetitiva.

Dimensión cognitiva: El trabajo de limpiar el ostión, es una actividad que implica destreza cognitiva, implica habilidades de precisión, coordinación viso-motriz, así como también de percepción, dada la adaptación a olores y texturas. Algunas trabajadoras refieren desagrado al trabajar con esa materia prima porque se les impregna el olor. Sin embargo, el aspecto más importante en el proceso de eviscerado, es definir la rapidez que se necesita para alcanzar las metas personales, ya que si es necesario las mujeres hacen un compromiso con su salud para producir lo necesario y así alcanzar el bono, por ejemplo : dejar de ir al baño y planear hacer la mayor parte de los bols por la mañana. Además, hacen un compromiso entre la percepción de calidad y la velocidad de trabajo. Ciertamente, el aumento de velocidad disminuye la precisión al sacar las vísceras del ostión.

Dimensión social : La relación es funcional, orientada a la obtención de materia prima suficiente para alcanzar el bono de producción. Dado que el incentivo es individual, se establecen relaciones de competencia entre las trabajadoras, por sobre las estrategias 
de colaboración colectiva. Por otra parte, es frecuente ver que las trabajadoras que tienen más experiencia, son las que tienen mayor productividad, por lo cual se han ganado el respeto de las demás. La experiencia, les ha permitido desarrollar estrategias para reducir el número de operaciones en la evisceración del ostión, lo que les otorga mayor margen de maniobra, siendo ellas las que menos se lesionan. No obstante, este saber-hacer no es valorado en los dispositivos de formación de la empresa, que se orientan a breves consignas sobre cómo hacer el trabajo.

Dimensión organizacional : La remuneración se basa en el salario mínimo, más un bono de producción individual, donde el rendimiento es evaluado por Kg de producción, que debe alcanzar la trabajadora para lograr la bonificación en cada turno. La primera escala de bonificación de la sección de eviscerado comienza en $36 \mathrm{Kg}$ con un bono de \$ 0.06 USD, la producción entre 0 a 35 kilogramos, no es bonificada, a diferencia de lo que ocurre aguas arriba en el puesto de desconche (donde la mayoría son hombres), allí la primera escala de bonificación es de 0 a $250 \mathrm{Kg}$ con bonificación de \$0,15 USD. Esto obliga a las trabajadoras ir a un ritmo intensificado, ya que ellas buscan hacer 12 bols o más por día.

Dentro de la sección de evisceración, las trabajadoras además deben llenar los bols con $6 \mathrm{~kg}$. de ostión. Si la trabajadora sobrepasa esa cantidad, la supervisora de esa sección transfiere esa cantidad excedente, a cualquier otra trabajadora, por lo tanto, pierde su producción.

El trabajo de las mujeres se valora menos, lo que se traduce en un bono de producción menor. Aunque producen menos peso, las trabajadoras realizan un trabajo muy minucioso y deben cumplir con criterios de calidad muy exigentes.

\subsubsection{Propuestas de soluciones}

A pesar de que el determinante principal, en la intensificación del trabajo, era existencia de bonos de producción individual, la propuesta de reestructurar el sistema de remuneración no fue aceptado por las trabajadoras y tampoco por la gerencia, lo que refleja la cultura del sector productivo, dado que uno de los argumentos era la posibilidad de que las trabajadoras emigren a otras empresas o regiones donde este sistema de remuneración, es el que predomina y permite a los trabajadores y trabajadoras alcanzar mayores salarios. Sin embargo, fue posible implementar un dispositivo de formación a partir del análisis en profundidad de las estrategias desarrollas por las trabajadoras más experimentadas, reconociendo su saber-hacer. También se mejoro el sistema de escaldado aguas arriba lo que impactaba en una menor exigencia al llegar en mejores condiciones la materia prima al puesto de eviscerado. También, se establecieron rotaciones de puestos de trabajo, pero la existencia del bono individual también limitó esta medida, así como también la implementación de pausas de descanso.

\subsection{Caso 2 : Operadores de descarga de pescado desde las bodegas de una embarcación (Empresa 2)}

\subsubsection{Contexto, demanda inicial y actividad}

La empresa donde se realiza el estudio lleva más de 40 años en la ciudad, fue la primera en instalarse en este territorio, donde la actividad pesquera industrial en esa zona era 
inexistente, lo que durante sus primeros 20 años represento la principal fuente de empleo en la ciudad. Hasta la crisis de la pesca en los años 90's, generada por la escasez de pesca atribuida en gran parte a los efectos causados por el fenómeno de la corriente del niño. Luego, la actividad pesquera de la región vuelve a comenzar, sustentada en gran parte por el trabajo que realizan los pescadores artesanales, la compañía establece una alianza estratégica con el Sindicato de Armadores de Lanchas Artesanales, para el abastecimiento de la materia prima, dada la paralización de la flota industrial que tenía la compañía, lo que vuelve a dar vida a la empresa, que actualmente se dedica a la producción de harina de pescado.

La demanda inicial surge desde el Departamento de Prevención de Riesgos, este solicita "Efectuar una intervención ergonómica del puesto de trabajo de Operador de Descarga para obtener un levantamiento objetivo de la actividad con el propósito de poder identificar los riesgos asociados y de qué manera se pueden mejorar". Lo que se requiere, es disminuir el número elevado de accidentes y de TME en la descarga de los peces.

El proceso de descarga de las embarcaciones de pesca, comienza una vez que estas atracan al costado del pontón de descarga, que es un artefacto naval, embarcación sin propulsión que se encuentra anclado en el mar en 4 puntas (Figura 3), que se encuentra ubicado a unos $800 \mathrm{~m}$ de distancia a la planta procesadora de harina de pescado.

Figura 3 : Ubicación del Pontón en relación a la planta procesadora de la harina de pescado

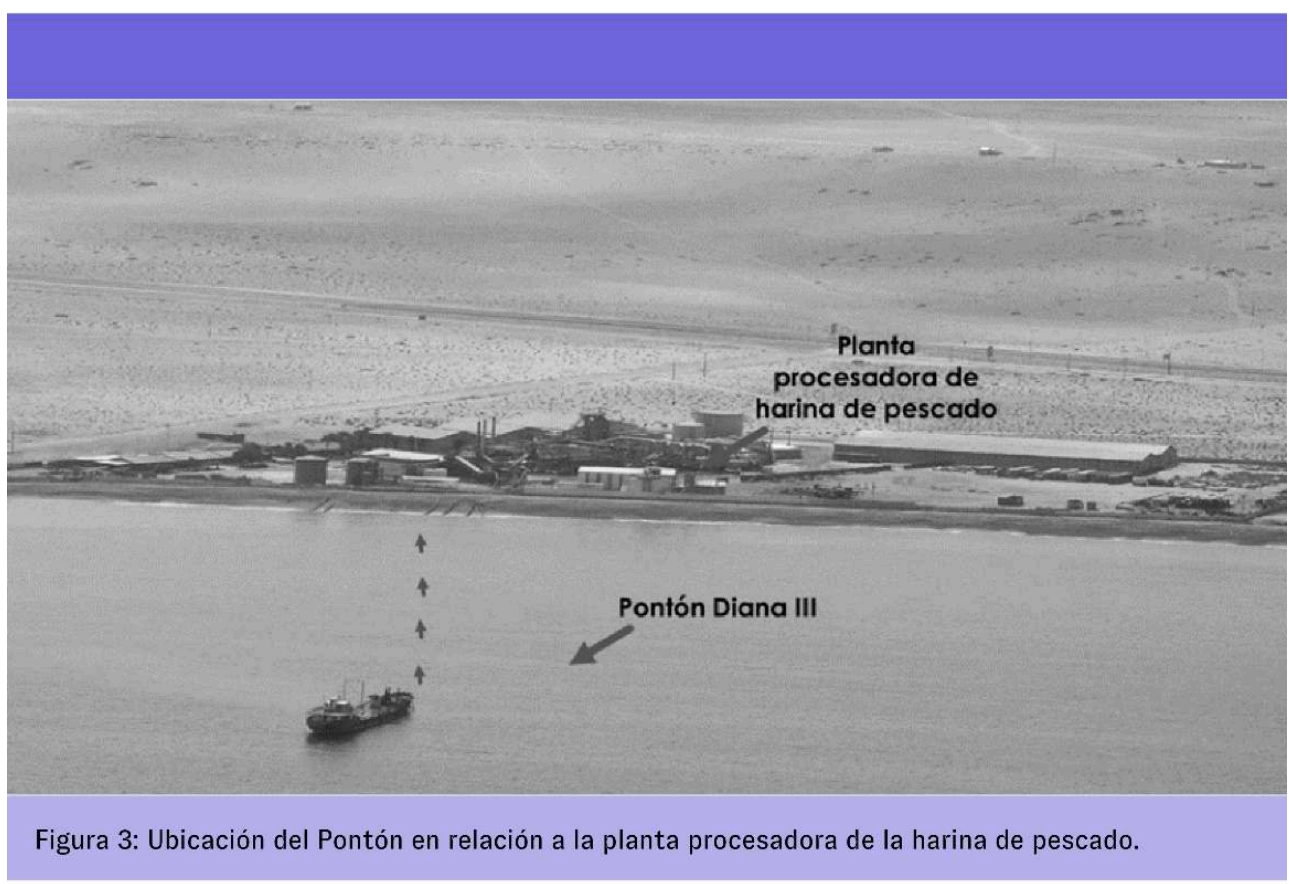

Figura 3 : Ubicación del Pontón en relación a la planta procesadora de la harina de pescado

El supervisor aborda la embarcación a fin de verificar en qué condición viene la carga y si es necesario que los trabajadores ocupen equipos de respiración de presión positiva o no. Una vez que se cuenta con la autorización correspondiente, se efectúa la inundación de las bodegas de las lanchas atracadas en el pontón y con ello la descarga de la pesca en dirección a la Planta procesadora a través de las tuberías de gran diámetro que se 
encuentran desde el pontón, bajo el mar, hasta las piscinas de la planta. Se pueden alcanzar 6720 toneladas por descarga, entre anchoveta y jurel.

La tarea de descarga puede tener una duración variable entre 30 a 60 min o más, en ese contexto existen varias etapas en las cuales el trabajador se encontrará dirigiendo el flujo de agua a alta presión con una manguera para la descarga que arroja agua salada a alta presión (02 Bar de presión). sobre cubierta y luego bajo cubierta dentro de la bodega de la embarcación para que sea succionado el producto por la "Yoma", que es una manguera de gran dimensión que se introduce en la embarcación, la cual actúa en forma similar a una aspiradora, por medio de la cual se succionan los pescados que se encuentran alojados en la embarcación y los traslada una distancia aproximada de 800 $\mathrm{m}$, desde el pontón a la planta procesadora.

A fin de mantener un mejor control de la dirección hacia donde se dirige el flujo de agua, el operador dobla la manguera constantemente efectuando fuerza mantenida en ciclos cortos durante la descarga. Al final de la descarga debe recoger las mangueras manualmente. A modo de ejemplo, se describen las operaciones de una parte de la tarea que representan una situación crítica, debido a que ocurre en la cruceta de las bodegas donde existe una altura que supera los $2.5 \mathrm{~m}$ de altura (Figura 4 ) :

1. Levanta el tronco, dejando manguera en una posición más cómoda.

2. Observa y desplaza de manguera con mano izquierda y derecha hacia su cuerpo

3. Se posiciona en el extremo de la manguera (pitón) con ambas manos.

4. Observa y ejerce presión sobre el pitón y dobla manguera.

5. Pasa el control del pitón, de la mano izquierda a su mano derecha.

6. Toma posición sentado y continua con descarga

Figura 4 : Trabajador Empresa 2 realizando descarga de los pescados desde la embarcación

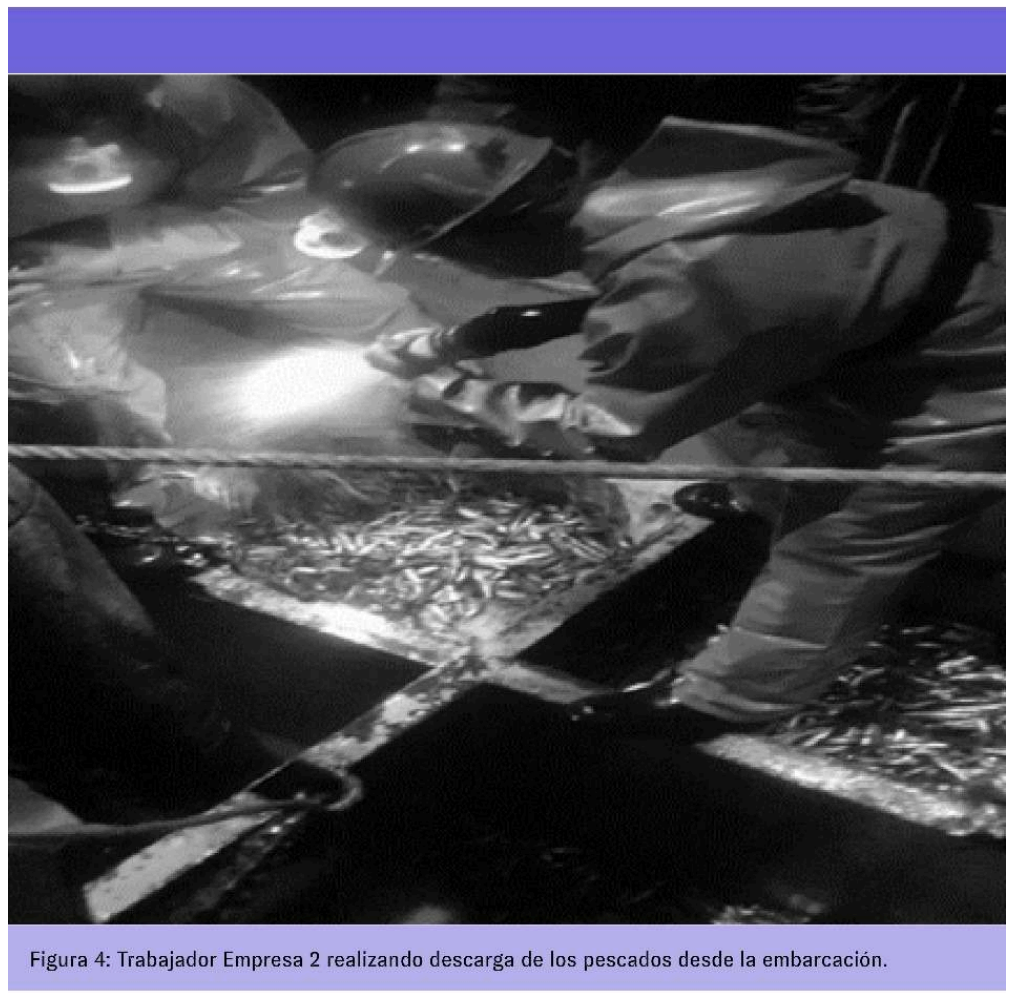

Figura 4 : Trabajador Empresa 2 realizando descarga de los pescados desde la embarcación 


\subsubsection{Prediagnóstico} para los trabajadores operadores de descarga. Las zonas más frecuentes de dolor referidas por las trabajadoras fueron los codos (33\%), muñecas (33\%) y hombros (15\%).

Figura 5 : Frecuencia porcentual del dolor referido por los trabajadores operadores de descarga (Empresa 2) según zona de dolor

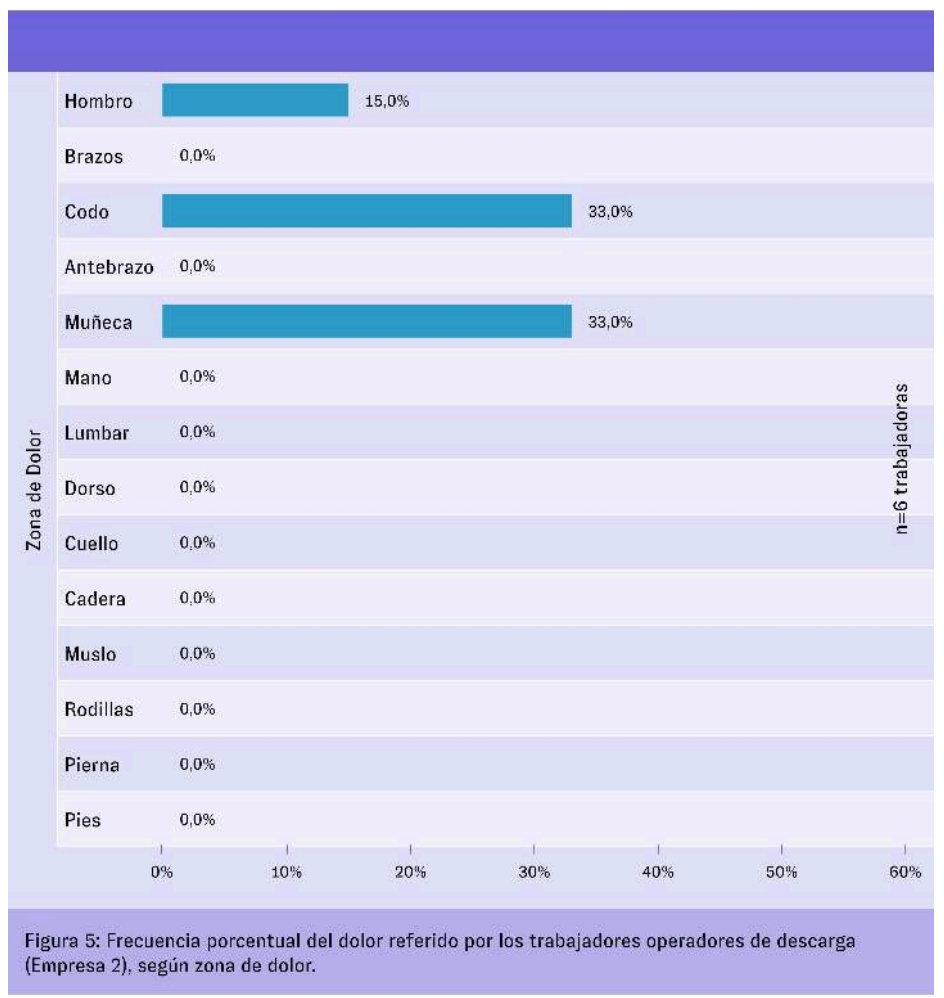

Figura 5 : Frecuencia porcentual del dolor referido por los trabajadores operadores de descarga (Empresa 2) según zona de dolor

Por otra parte, existen problemas de seguridad en la descarga que se suscitan cuando la pesca de una embarcación lleva más de $24 \mathrm{~h}$ en sus bodegas acumulando ácido sulfhídrico por la descomposición de los pescados. En estos casos la descarga se realiza de una forma diferente, ocupando medios de aire a presión positiva, a fin de evitar la intoxicación de los trabajadores. En algunas ocasiones los trabajadores omiten este paso y realizan las descargas sin sus equipos de protección, cuando no hay control del supervisor. Además, en ocasiones no hay ocupación de elementos de fijación, aun cuando las bodegas superan los $2.3 \mathrm{~m}$. de altura, esto si bien está en la "tarea prescrita" de los trabajadores, en la realidad no siempre están disponibles para los trabajadores.

Los trabajadores refieren que para "no ser menos" o considerados "débiles" ante sus pares, asumen estos riesgos, también refieren estar "habituados" a realizar la actividad de este modo a través de los años. No obstante, también hay ausencia de instrucción y capacitación de los riesgos a los cuales se encuentran expuestos

Variabilidad del producto: Los productos que son procesados en la industria, corresponden al Jurel y la Anchoveta, esta última es la más extraída en el territorio donde se emplaza la empresa, también es la que genera mayor dificultad de manejo de

Laboreal, Volume 17 №2 | 2021 
la descarga. La anchoveta en un pez de dimensiones pequeñas (similar al tamaño de un lápiz), al ser tan pequeño, en una embarcación se comporta como el agua, por eso la faena de descarga debe ejecutarse de una manera segura, evacuando las bodegas en forma simétrica a fin de evitar la "escora" de la embarcación, que es la posibilidad que se dé vuelta y producir un accidente de gran magnitud, como por ejemplo que la embarcación se hunda.

Aspectos colectivos del trabajo: El trabajo de descarga se realiza entre dos operadores, los cuales se ponen de acuerdo antes de subir a la embarcación, identificando claramente cuáles serán sus bodegas a descargar. Ambos trabajan en primera instancia sobre la cruceta de la embarcación (espacio superior de las bodegas, ver Figura 4) inundando las bodegas y posterior a ello, se introducen al interior de las mismas, esta labor debe ser coordinada, la cual realizan a viva voz, una descarga mal ejecutada puede causar la "escora" de la embarcación, es por esto que la comunicación entre ambos debe ser fluida.

\subsubsection{Descripción de las limitaciones, exigencias y riesgos asociados a la actividad}

Dimensión física: Es una exigencia importante en la actividad, asociada a la repetitividad, el uso de fuerza que es referida por los trabajadores como importante (sobre 3 puntos en la Escala de Borg) para mantener el control de la manguera con agua que sale a alta presión. Como se puede ver en la Figura 6, el esfuerzo físico está presente en toda la descarga desde la instalación de las mangueras en cubierta de la nave a descargar, como al retiro de estas. También hay carga postural estática que es frecuente, en extremidades superiores al sostener las mangueras, pero también en las posturas de flexión mientras se equilibra sobre las crucetas en cubierta y luego al interior de la bodega. Por otra parte, el trabajo es exigente en cuanto a la precisión de los gestos, para direcciona el flujo de agua para realizar la descarga de las bodegas de manera rápida y eficiente.

41 Las dimensiones de las bodegas de lancha son un determinante considerable para el trabajador, producto que están diseñadas para alojar peces al interior, pero no para la actividad de descarga, con espacios reducidos en cubierta que disminuyen la movilidad del trabajador, sin apoya manos, ni superficies adecuadas para apoyar los pies. Es así como, los trabajadores adoptan posturas extremas para poder descargar los productos antes mencionados, arriesgando en muchas oportunidades su integridad física. 
Figura 6 : Gráfico de tiempo, operaciones al manipular la manguera en un ciclo de descarga de una lancha, según lugar de la embarcación

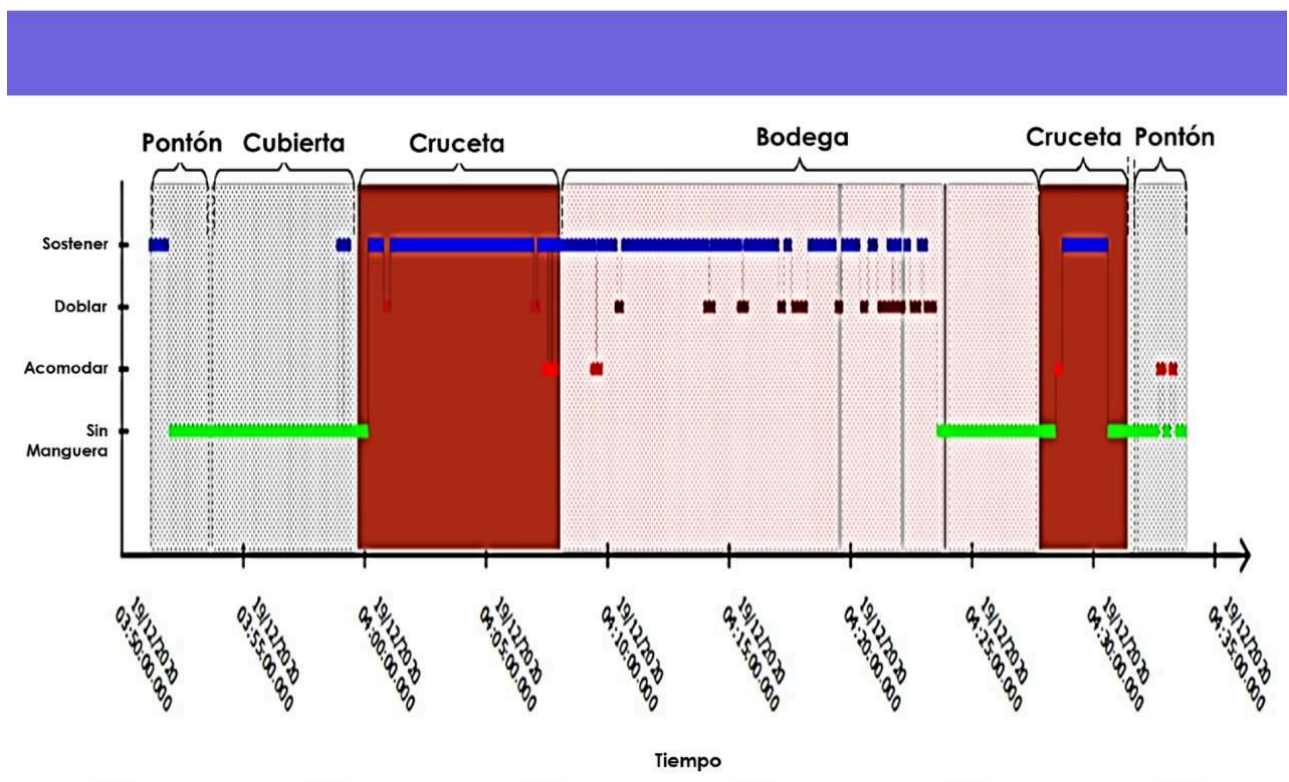

Figura 6: Gráfico de tiempo, operaciones al manipular la maguera en un ciclo de descarga de una lancha, según lugar de la embarcación.

Figura 6 : Gráfico de tiempo, operaciones al manipular la manguera en un ciclo de descarga de una lancha, según lugar de la embarcación

Dimensión cognitiva: La actividad de descarga utilizando una manguera para la inundación, descarga y limpieza de las bodegas genera una exigencia cognitiva constante para el operador, dado que este debe estar en constante atención de las condiciones que varían a cada minuto, para no resbalar y caer al interior de las bodegas, para evitar golpearse con la "yoma, mantener el control de la dirección hacia donde se dirige el flujo de agua, evitando el riesgo de que los pescados se acumulen en un solo lado de la embarcación (escore), lo que puede generar un accidente. A esto se agrega la dificultad del trabajo en el mar con el movimiento de la embarcación, el trabajo de noche con escasa luz (solo linternas) y la exigencia temporal, en cuanto a que la descarga se realice en el tiempo previsto (30 a $60 \mathrm{~min}$ ).

Dimensión social: La relación entre los trabajadores es funcional, orientada a la obtención de los resultados del proceso productivo, referida por los trabajadores como "un buen ambiente de trabajo". Los operadores de descarga tienen bajos niveles de escolaridad, el conocimiento sobre el oficio es transmitido desde los trabajadores más antiguos a los más novatos, un aspecto que es característico en el rubro de la pesca artesanal. Este saber-hacer no es valorado en los dispositivos de formación de la empresa, que se orientan a breves consignas sobre cómo hacer el trabajo. Existe una cultura de trabajo familiar, donde habitualmente hay distintas generaciones de familiares que han desarrollado este mismo trabajo, y con lo cual han aprendido el oficio. Siempre son hombres quienes realizan este trabajo, existiendo conductas que ellos refieren como "propias de hombres de mar", los cuales deben ser personas "fuertes", suelen minimizar los riesgos a los que se encuentran expuestos ya sea demostrar esto ante sus pares, normalizando los riesgos del trabajo, no usando elementos de 
protección personal o verbalizando que el trabajo, es liviano y que no genera mayores dolores.

Dimensión organizacional: La actividad se realiza en lo que en Chile se denomina "trabajadores portuarios eventuales", primando estas normas por sobre las reglas generales en materia de contratación, jornada, remuneración y término de contrato (Carrillo \& Santander, 2005). Esta labor es realizada por dos Operadores de Descarga por cada embarcación. Los turnos están compuestos de tres personas y la empresa posee dos turnos con una duración de $8 \mathrm{hrs}$ diarias, uno diurno y otro nocturno, pero generalmente las descargas se realizan a contar de las $16: 00 \mathrm{hrs,} \mathrm{en} \mathrm{adelante} \mathrm{hasta} \mathrm{la}$ madrugada, por lo cual el trabajo se concentra en la tarde y noche.

La remuneración es fija, pero cuando existe la temporada alta de operación, la cual suele ser generalmente 6 meses en el año, los operadores pueden doblar su salario, efectuando descargas con jornadas prolongadas y con bonos de producción a partir de la cantidad de toneladas de pescado descargado por cada turno. Los trabajadores pueden pasar semanas completas, con jornadas que en ocasiones sobrepasan el límite de las $10 \mathrm{hrs}$. Por otra parte, no existe una cadena de mando establecida en la plataforma de descarga (pontón), producto que todos los que realizan alguna labor dentro de ella, pasaron por el puesto de operador de descarga. El supervisor trabaja en el muelle distante uno $800 \mathrm{~m}$ de la plataforma y la comunicación es en forma radial.

\subsubsection{Propuestas de soluciones}

Se recomendó incorporar la adquisición de un pitón que cuente con una válvula de corte rápido y de peso ligero, que se acomode a la actividad de descarga por los operadores. Incorporar como medida preventiva una rejilla que se aloje sobre la cruceta de las bodegas, la cual permita el paso del agua al interior de las mismas y evite la posible caída de los trabajadores al interior de las bodegas. Incorporar en las embarcaciones un manifold de inundación, que se pueda controlar desde cubierta, evitando la exposición prolongada de los trabajadores al interior de espacios confinados (bodegas).

\section{Discusión}

47 A finde mostrar las realidades en que se encuentran hombres y mujeres en diferentes emplazamientos productivos, dentro de un mismo territorio, podemos decir la actividad de trabajo se encuentra marcada por las inequidades, en primer lugar, dada las restringidas posibilidades presentes para acceder a la ocupación que existen en esa localidad, tanto hombres como mujeres, deben realizar un compromiso con su salud y seguridad para realizar las actividades descritas. Por una parte, las mujeres en la empresa procesadora de ostiones despliegan un tipo de actividad intensificada a causa de los incentivos a la producción. "Las mujeres se distinguen por su energía, su habilidad en los trabajos que exigen una sensibilidad motriz muy grande, movimientos rápidos y precisos" (Frois, 1926, en Hirata 1997, p. 218), en este aspecto, muestran una habilidad especial para la materia productiva en que se desarrollan. Sin embargo, las trabajadoras en ese contexto no cuentan con posibilidades de desarrollo mayores, en este sentido, la empresa significa para ellas forma de sobrevivencia, en un lugar en que no existen muchas ofertas laborales, que no impliquen emigrar hacia otras ciudades. 
Frente a la conciencia acerca de la movilidad salarial, la empresa entrega incentivos de producción que son atractivos para las trabajadoras, "La mujer es, por otra parte, ahorrativa, previsora por naturaleza y aun codiciosa, una leve ganancia basta a veces, para hacerla trabajar más allá de sus fuerzas" (Frois, 1926, en Hirata 1997, p. 218).

Así mismo, las mujeres en la empresa procesadora de ostiones realizan una actividad de trabajo de eviscerado, la cual es poco valorada por la empresa y al contar con condiciones de remuneración poco equitativas, ya que el interés empresarial está en explotar las materias primas en el territorio, genera efectos negativos para la salud de estas trabajadoras, como así lo muestran la alta prevalencia de TME y la fatiga en el trabajo, y a la vez impactan en el ambiente con la extracción intensificad de estas materias primas.

Por otra parte, los operadores de descarga de pescado, en la otra empresa, deben adaptarse a condiciones ambientales, temporales, dimensiones físicas del puesto de trabajo, las cuales contrastan con el trabajo repetitivo-estático que resalta en las evisceradoras de ostiones, ya que el trabajo de los operadores, es un trabajo de exigencia física elevada, con la exposición frecuente a posturas forzadas y riesgos químicos, lo cual impone centralmente otro tipo de determinantes, como a nivel de dispositivos técnicos, etc. Sin embargo, llama la atención el que se piense al trabajador como en un obrero y no se considere lo extenuante que puede llegar a significar la jornada para estos trabajadores, ya que la empresa exige a los fuertes y hábiles cuerpos de estos trabajadores que sirvan a la empresa para la extracción total del pescado, tal como lo hiciera una máquina. Uno se pregunta, es este tipo de empresas, donde la acción humana es tan importante, ¿Cómo poder hacer avanzar más en las representaciones acerca de la salud y la seguridad que tienen, tanto encargados como los propios trabajadores, acerca de la actividad de trabajo? ¿Cómo hacer que comprendan que las condiciones de trabajo no son espontáneas de la naturaleza, son dispuestas por la empresa? El trabajo, pese a todo lo que puede significar para las personas, satisfacción, ingresos, identidad, etc., es importante que no enferme a los trabajadores, para que desarrollen actividades productivas con agrado y en ausencia de miedos, para desarrollar valor.

En ambas actividades presentadas, las empresas desconocen el valor que tiene la destreza física para actuar, las destrezas cognitivas para conocer la materia con la que se trabaja y su naturaleza, es un conocimiento que tempranamente desarrollan los(as) trabajadores(as) en estos lugares, incluso en algunos casos se inicia en los hogares con el aprendizaje observacional que se desarrolla alrededor las vivencias y al traspasar el saber-hacer de una generación a otra. Este conocimiento, se aprovecha poco para hacer desarrollar los procesos de mejora continua, lo cual aportaría para desarrollo de tecnologías dentro de la empresa. Sumado a lo anterior, en las empresas, poseen representaciones acerca de la Salud y la Seguridad del trabajo que son propias de las actividades de tipo artesanal, asociada a una filosofía de que las cosas deben ser y hacerse de un modo siempre y esperando que se mantengan así los procesos productivos.

51 En una intervención ergonómica que busca responder a la petición de una empresa cuyo interés en la prevención (de origen normativo) de riesgos de salud y seguridad en el trabajo a menudo es nuevo y frágil. El interés en las cuestiones de género, por lo general es nulo (Astudillo et al., 2019). Sin embargo, el análisis ergonómico ayuda en la comprensión del trabajo y sus inequidades. Así como en la problemática de género, 
inherente en los contextos de trabajo a causa de la división sexual del trabajo, prevalece una organización del trabajo basados en obsoletas prácticas de producción de bienes y servicios, donde el trabajo a destajo puede parecer arcaico (Premji, Lippel, \& Messing, 2008), y que por ejemplo están en oposición a combatir, los riesgos psicosociales. Desde este punto de vista, y retomando el párrafo anterior, la relación que tienen los incentivos a la producción con el deterioro de la SST, se vincula con los efectos en las relaciones interpersonales que esto provoca. Pero si detallamos más, se encuentran inequidades de género en la escala de bonificación, ya que las mujeres en eviscerado deben hacer mayor cantidad de producto (ostión eviscerado) comparativamente al caso de los hombres de desconchado, lo cual muestra que deben hacer más esfuerzo. Por otro lado, los trabajadores de la embarcación, si bien el trabajo de ellos es diferente, pueden acceder a una buena remuneración, ya que están en el inicio de la línea productiva y es más valorado ese trabajo desde la perspectiva de la división sexual del trabajo.

Dentro de los trabajadores es frecuente oír expresiones de frustración experimentada a partir del trabajo, que a veces no se puede hacer bien, a causa de la intensificación de la actividad y no poder lograr una óptima performance, genera mayor individualismo y distanciamiento en la relación entre él y los colectivos de trabajadores, debilitándose la comunicación y produciendo escaso diálogo entre trabajadores y empleadores.

Para las mujeres del eviscerado, incorporarse a trabajar en la empresa de procesamiento de ostiones simboliza aspectos relacionados con el conocimiento sobre el trabajo a nivel familiar, que se traspasan a las generaciones, en el cual se observa que "El territorio va a contener las prácticas sociales y los sentidos simbólicos que los seres humanos desarrollan en la sociedad en su íntima relación con la naturaleza" (LlanosHernández, 2010, p. 208); en este caso, la integración a este trabajo va a ser para obtener recursos para subsistir, para pagar los estudios de hijos y disponer recursos para el hogar, ya que este tipo de actividad no es desarrollada por gusto entre las trabajadoras. Sin embargo, una vez iniciadas en el trabajo ellas son capaces de desarrollar un conocimiento y un saber-hacer que las hace conocedoras de la materia con la cual trabajan, al punto de generar conocimiento respecto de la elección de herramientas para el uso diario en la actividad de trabajo en el caso de las trabajadoras del caso uno, es un elemento determinante en el cuidado de la salud y de la seguridad en el trabajo.

Sin el conocimiento desarrollado a partir del conocimiento del territorio y de la materia prima, el trabajo pierde continuidad en el tiempo y en el espacio de una sociedad. El territorio ayuda en la interpretación y comprensión de las relaciones sociales vinculadas con la dimensión espacial; va a contener las prácticas sociales y los sentidos simbólicos que los seres humanos desarrollan en la sociedad en su íntima relación con la naturaleza, algunas de las cuales cambian de manera fugaz, pero otras se conservan adheridas en el tiempo y el espacio de una sociedad (Llanos-Hernández, 2010).

Nuestra sociedad, promueve trabajos en los cuales las personas entreguen sus habilidades para obtener mayores utilidades a los menores costos productivos, de ese modo, "hoy sobre todo el trabajo obrero femenino se caracteriza por una mayor precariedad, una mayor flexibilidad que la de los varones...La división sexual del trabajo sigue existiendo y contribuye a la gestión de la mano de obra, a la modernización de la economía" (Hirata et al., 1997, p. 223), lo cual se refleja en que el 
trabajo que generan las mujeres de la sección de eviscerado de ostiones, es de alto valor, ya que este llega a la exportación de estos productos que son apreciados a nivel internacional y contribuyen a entregar una fuente laboral a un porcentaje importante de la población de ese territorio. Por otra parte, los trabajos derivados de la pesca artesanal son en general determinados por temporadas, ya sea de cosecha para los ostiones, como cuotas de pesca en la industria de la harina, no requieren estudios previos de sus actores, si no habilidades las que generalmente son heredadas por sus padres o parientes cercanos, lo que concuerda con otras investigaciones (Major, Clabault, \& Wild, 2021 ; Ullilen-Marcilla et al., 2018 ; Major \& Vézina, 2017).

La zona geográfica donde se emplazan las empresas, es propicia para el desarrollo de esta actividad, debido que cuenta con bahías calmas, para el desembarco de pescado y aguas nutritivas para el filtrado de los ostiones a diferencia de otras pertenecientes a la misma zona geográfica. El territorio determina el puesto de trabajo al que puede optar una persona de acuerdo a sus posibilidades de desarrollo, educación y también el acceso a remuneración, que en estos dos casos son pagados con bonificación durante los periodos estacionales en que la producción es aumentada, aprovechando las condiciones de explotación de la materia prima. Estas empresas, independiente la una de la otra, comparten prácticas propias del sector y arraigadas en el territorio donde se emplazan, de hecho, en ambos casos los incentivos al trabajo individual, la escasa cultura de prevención de los riesgos laborales y por otra parte el trabajo artesanal donde el conocimiento generado, el saber-hacer de la actividad es transmitido entre familiares y cercanos que también habitan el territorio.

Las condiciones precarias en el ámbito laboral son determinadas por la temporalidad de las faenas y la informalidad, las cuales no permiten la generación de oportunidades laborales estables, que permitan un ingreso digno para la sustentación de sus familias, lo que representan determinantes sociales de la salud de los trabajadores y trabajadoras (Solar \& Irwin, 2011). Tanto la actividad pesquera como la acuicultura presentan altos niveles de informalidad laboral que afectan de manera particular a las mujeres, y principalmente a las jefas de hogar. Esta informalidad se refleja no solo en los altos porcentajes de trabajo eventual que enfrentan, sino también en su limitado acceso a la seguridad y protección social, baja asociatividad y limitado acceso a bienes y servicios para el desarrollo de su actividad pesquera o acuícola (Valdés, Rebolledo, Pavez, \& Hernández, 2014).

\section{Conclusiones}

El análisis de la actividad y el proceso de construcción participativa generaron resultados, aunque limitados, particularmente a aquellos determinantes ligados a los dispositivos técnicos, formación y pausas de trabajo, entre otros. Los determinantes más importantes en las exposiciones a riesgo, como la intensificación del trabajo producto de los salarios con bonificaciones individuales, no fue posible modificar, y en ambos casos, independiente de la empresa, el contexto de la intervención ergonómica (la única realizada en ambas empresas) no fue suficiente para aquello. Se podría afirmar que estas intervenciones permitieron generar cambios de representación sobre la SST en los actores del medio de trabajo, no obstante, la pertenencia a un sector cuya cultura de SST es frágil, no garantiza la sustentabilidad de las intervenciones. 

de ingresos y ellas están dispuestas a trabajar de todos modos, realizando un compromiso con su salud si es necesario, a fin de lograr sus objetivos personales, como sacar adelante a sus familias, por lo cual el territorio marca fuertemente sus trayectorias laborales y de vida. hay una baja valorización de la salud de la persona, pero en un sentido más amplio del territorio, esta realidad no es diametralmente diferente al sector económico de la pesca en los más $4000 \mathrm{~km}$ de extensión que esta tiene en Chile.

Ahora bien, teniendo en cuenta la naturaleza del trabajo dentro de este contexto territorial independiente de aquellos se refleja en ambas intervenciones como el trabajo esta segmentado entre hombres y mujeres, determinado por la división sexual de la producción, que hace a las mujeres ocupar los espacios determinados dentro una planta procesadora al interior de la cadena productiva, realizando actividades minuciosas y de habilidad motriz fina y en el caso de los descargadores de pescado, en el inicio de la cadena productiva realizando el trabajo que implica el uso de la fuerza y la exposición a riesgos de accidentes (Astudillo \& Ibarra, 2014 ; Valdés et al., 2014). culadas con la dimensión espacial (Llanos-Hernández, 2010 ; Deambrosi \& Tagliabue, 2013); las personas que trabajan en estas empresas, emplazadas en este territorio comparten un saber-hacer que no es posible de encontrar con facilidad en otros territorios. Han tenido un vínculo con la actividad de trabajo, conociendo de la actividad por sus propias familias que ya estaban en interacción con la actividad en este mismo territorio. Fuera de la identificación familiar que puede identificar con fuerza a algunas familias con esta actividad, también existen personas que tienen sólo una identificación funcional con la actividad, como un medio para obtener dinero.

Ambas empresas, en el territorio, representan para las personas su única fuente estable trayectorias laborales y de vida.

\section{BIBLIOGRAFÍA}

Astudillo, P., \& Ibarra, C. (2019). Os incentivos à produção, seus efeitos na segurança e na saúde do trabalho e entre coletivos de trabalho : uma abordagem da formação em ergonomia. Laboreal, 15(1), 1-22. https://doi.org/10.4000/laboreal.1393

Astudillo, P., \& Ibarra, C. (2014). La perspectiva de género, Desafíos para la Ergonomía en Chile : Una revisión Sistemática de Literatura. Ciencia \& Trabajo, 16(49), 28-37. http://dx.doi.org/10.4067/ S0718-24492014000100006

Caroly, S., Major, M., Probst, I., \& Molinié, A. (2013). Le genre des troubles musculo-squelettiques : Interventions ergonomiques en France et au Canada. Travail, genre et sociétés, 29, 49-67. https:// doi.org/10.3917/tgs.029.0049

Carrillo, I., \& Santander, A. (2017). Modernización portuaria en Chile. Síntesis tecnológica, 2(2), 63-68. 
Castellucci, H., Viviani, C., Hernández, P., Bravo, G., Martínez, M., Ibacache, J., \& Bartsch, A. (2021). Developing countries and the use of ISO Standard 11228-3 for risk management of WorkRelated Musculoskeletal Disorders of the Upper Limbs (WRMSDs-ULs) : The case of Chile. Applied Ergonomics 96, 103483. https://doi.org/10.1016/j.apergo.2021.103483

Deambrosi, N., \& Tagliabue, P. (2013). Territorio, trabajo y salud : Una mirada desde dos actividades productivas. Producción granífera en Zona Núcleo y producción ladrillera en el sudeste santiagueño. Universidad Nacional de la Plata. Facultad de Humanidades y Ciencias de la Educación. Geograficando, 9(9), 1-11.

Guérin, F., Laville, A., Daniellou, F., Duraffourg, J., \& Kerguelen, A. (2008). Comprendre le travail pour le transformer : la pratique de l'ergonomie. Toulouse : ANACT.

Hirata, H., Kergoat, D., \& Zylberberg-Hocquard, M. H. (1997). La división sexual del trabajo : Permanencia y cambio. Buenos Aires : Asociación Trabajo y Sociedad.

Ibarra C., \& Astudillo P. (2021). Manual Handling Task of Bovine Quarters Among Delivery Operators in a Chilean Slaughterhouse Company : A Case Study with Ergonomic Approximation. In N. Black, P. Neumann, I. Noy (Eds.), Proceedings of the 21st Congress of the International Ergonomics Association (IEA 2021). IEA 2021. Lecture Notes in Networks and Systems (pp. 208-216). Springer. https://doi.org/10.1007/978-3-030-74608-7_27

Instituto Nacional de Estadísticas de Chile (2021). Banco de datos de la Encuesta Nacional de Empleo. Consultado junio, 07, 2021 : http://bancodatosene.ine.cl/

Llanos-Hernández, L. (2010). El concepto del territorio y la investigación en las ciencias sociales. Agricultura, sociedad y desarrollo, 7(3), 207-220.

Major, M-E., \& Vézina, N. (2017). The Organization Of Working Time : Developing An Understanding And Action Plan To Promote Workers' Health In A Seasonal Work Context. New Solutions : A Journal of Environmental and Occupational Health Policy, 27(3), 403-423. https://doi.org/ $10.1177 / 1048291117725712$

Major, M-E., Clabault, H., \& Wild, P. (2021). Interventions for the prevention of musculoskeletal disorders in a seasonal work context : A scoping review. Applied Ergonomics, 94, 103417. https:// doi.org/10.1016/j.apergo.2021.103417

Major, M-E., \& Vézina, N. (2011). Élaboration d'un cadre de référence pour l'étude des stratégies : analyse de l'activité et étude de cas multiples dans deux usines de crabe. Perspectives interdisciplinaires sur le travail et la santé, 13(2). https://doi.org/10.4000/pistes.1843

Major, M-E., \& Vézina, N. (2015). Analysis of worker strategies : a comprehensive understanding for the prevention of work-related musculoskeletal disorders. International Journal of Industrial Ergonomics, 48, 149-157. https://doi.org/10.1016/j.ergon.2015.05.003

Messing, K. (2000). La Santé des travailleuses : la science est-elle aveugle? Montréal : Éditions du remue-ménage.

Messing, K., Lefrançois, M., \& Saint-Charles, J. (2018). Observing Inequality : Can Ergonomic Observations Help Interventions Transform the Role of Gender in Work Activity? Computer Supported Coop Work, 30, 215-249. https://doi.org/10.1007/s10606-018-9337-X

Premji, S., Lippel, K., \& Messing, K. (2008). “ On travaille à la seconde ! » Rémunération à la pièce et santé et sécurité du travail dans une perspective qui tient compte de l'ethnicité et du genre. Perspectives interdisciplinaires sur le travail et la santé, 10(1). https://doi.org/10.4000/pistes.2181

Solar, O., \& Irwin, A. (2007). Towards a conceptual framework for analysis and action on the social determinants of health. Geneva : WHO Commission on Social Determinants of Health. 
St-Vincent, M. Vézina, N., Bellemare, M, Denis, D., Ledoux, E., \& Imbeau D. (2011). L'intervention en ergonomie. Éditions MultiMondes et IRSST.

Superintendencia de Seguridad Social de Chile (2020). Informe Estadísticas de Accidentabilidad 2020. SUSESO 2020. Consultado junio, 07, 2021 : https://www.suseso.cl/608/w3propertyvalue-59606.html

Valdés, X., Rebolledo, L., Pavez, J., \& Hernández, G. (2014). Trabajos y familias en el neoliberalismo hombres y mujeres en faenas de la uva, el salmón y el cobre. Cuadernos del CENDES, 32(89), 257-260.

Vézina, N. (2001). La pratique de l'ergonomie face aux TMS : ouverture à l'interdisciplinarité. In Proceedings of SELF-ACE Conference. Montréal.

Ullilen-Marcilla, C., \& Ullilen-Marcilla, R. (2018). Prácticas desarrolladas por los trabajadores con predominio del trabajo manual : caso de estiba de productos pesqueros. Laboreal, 14(2). https:// doi.org/10.4000/laboreal.685

\section{RESÚMENES}

La actividad de la pesca, produce identificación y conocimientos del oficio en un contexto dado. En este artículo se presentan 2 estudios de casos : 1) Trabajadoras en proceso de transformación de ostiones, 2) Operadores de descarga de pescado, en otra empresa pesquera. Ambas situaciones se analizan desde el modelo centrado en la persona en actividad, los cuales se llevaron a cabo mediante el esquema de la intervención ergonómica, presentando las siguientes etapas de la intervención: Análisis de la demanda; Prediagnóstico; y Propuesta de la intervención. Las actividades estudiadas, muestran las inequidades vinculadas a los determinantes de la actividad, como la organización del trabajo, el pago mediante bonos de producción, el trabajo temporal. Ambas empresas, en el territorio, generan el desarrollo de conocimientos y habilidades que son transmitidas en generaciones. representan para las personas la única fuente estable de ingresos, pero llevándolas a realizar un compromiso con su salud.

A atividade pesqueira produz a identificação e o conhecimento do comércio num determinado contexto. Neste artigo são apresentados dois estudos de caso: 1) Mulheres trabalhadoras em processo de transformação de ostras ; 2) Operadoras de descarregamento de pescado, numa outra empresa pesqueira. Ambas as situações são analisadas a partir do modelo centrado na pessoa em atividade, as quais foram realizadas através do esquema de intervenção ergonómica, apresentando as seguintes etapas da intervenção: análise da demanda; pré-diagnóstico; e proposta de intervenção. As atividades estudadas mostram as desigualdades vinculadas aos determinantes da atividade, como a organização do trabalho, o pagamento por meio de bónus de produção, o trabalho temporário. Ambas as empresas, no território, geram o desenvolvimento de conhecimentos e competências que são transmitidos de geração em geração. Elas representam a única fonte estável de rendimento para as pessoas, mas levando-as a se comprometerem com a sua saúde.

L'activité de pêche produit l'identification et la connaissance du métier dans un contexte donné. Dans cet article 2 études de cas sont présentées: 1) Travailleuses dans le processus de transformation des huîtres, 2) Opérateurs de déchargement de poisson, dans une autre entreprise de pêche. Les deux situations sont analysées à partir du modèle centré sur la personne en activité, qui a été réalisé à travers le schéma d'intervention ergonomique, présentant les étapes suivantes de l'intervention: Analyse de la demande; Prédiagnostic; et Proposition de l'intervention. Les activités étudiées montrent les inégalités liées aux déterminants de l'activité, 
tels que l'organisation du travail, le paiement par les primes à la production, le travail temporaire. Les deux entreprises, sur le territoire, génèrent le développement de savoirs et de compétences qui se transmettent de génération en génération. ils représentent la seule source stable de revenus pour les personnes, mais les amènent à s'engager pour leur santé.

Fishing activity produces identification and knowledge of the trade in a given context. In this article two case studies are presented: 1) Women workers in the process of transformation of oysters ; 2) Fish unloading operators, in another fishing company. Both situations are analyzed from the model centered on the person in activity, which were carried out through the ergonomic intervention scheme, presenting the following stages of the intervention : analysis of the demand; pre-diagnosis; and proposal of the intervention. The activities studied show the inequities linked to the determinants of the activity, such as the organization of work, payment through production bonuses, temporary work. Both companies, in the territory, generate the development of knowledge and skills that are transmitted in generations. they represent the only stable source of income for people, but leading them to make a commitment to their health

\section{ÍNDICE}

Palabras claves: análisis de la actividad, territorio, intervención ergonómica, género, productos del mar

Keywords: analysis of activity, territory, ergonomic intervention, gender, seafood products

Palavras-chave: análise de atividade, território, intervenção ergonómica, género, mariscos

Mots-clés: analyse d'activité, territoire, intervention ergonomique, genre, produits de la mer

\section{AUTORES}

\section{PAMELA ASTUDILLO}

https://orcid.org/0000-0002-1457-2072

Programa de Ergonomía Ocupacional, Departamento de Kinesiología de la Facultad de Ciencias de la Salud, Universidad de Atacama,

Av. Copayapu 2872, Copiapó-Chile, código postal : 1533722

pamela.astudillo@uda.cl

\section{CARLOS IBARRA}

https://orcid.org/0000-0002-2178-6212

Programa de Ergonomía Ocupacional, Departamento de Kinesiología de la Facultad de Ciencias de la Salud, Universidad de Atacama, Av. Copayapu 2872, Copiapó-Chile, código postal : 1533722 carlos.ibarra@uda.cl

\section{FRANCISCO VALDÉS}

https://orcid.org/0000-0002-2711-429X

Estudiante Diplomado Ergonomía Ocupacional, Universidad de Atacama, Av. Copayapu 2872, Copiapó-Chile, código postal : 1533722

francisco.valdes.19@alumnos.uda.cl 